\title{
LA CONSTRUCCIÓN DE INDICADORES COMO PROBLEMA EPISTEMOLÓGICO
}

\section{THE CONSTRUCTION OF INDICATORS AS AN EPISTEMOLOGICAL PROBLEM}

Dr. Daniel Gutiérrez (dcgutier@sociales.uson.mx) División de Ciencias Sociales, Universidad de Sonora (Sonora, México)

\section{Abstract}

The construction of indicators does not constitute a smaller epistemological problem, quite the opposite, requires the exigency of a critical way to reflection, to take control and to reconstruct the reality; it requires of monitoring, and permanent ruptures and its corresponding forms to reorganize the thought. The main problem in the construction of indicators does not have to do with the theory itself or the operation of the concepts. The fundamental question is how someone thinks, how someone takes control and how the object is constructed. The indicators constitute an empirical definition of the object and are product of a theoretical construction of the subject on the phenomenon to discover specific concrete of the object.

Keywords: subject-object, epistemological ruptures, indicator, theoretical construction.

\section{Resumen}

La construcción de indicadores no constituye un problema epistemológico menor, todo lo contrario, requiere de la exigencia de un modo crítico de reflexionar, de apropiarse y reconstruir la realidad; requiere de vigilancia, y permanentes rupturas y sus correspondientes formas de reorganizar el pensamiento. El problema de fondo en la construcción de indicadores no tiene que ver con la teoría misma o con la operación de los conceptos. La cuestión fundamental es cómo se piensa, cómo se apropia y cómo se construye el objeto. Los indicadores constituyen una definición empírica del objeto y son producto de una construcción teórica del sujeto sobre el fenómeno para descubrir lo específico concreto del objeto.

Palabras clave: sujeto-objeto, ruptura epistemológica, indicador, construcción teórica.

\section{Introducción}

La cuestión de los indicadores en ciencias sociales constituye un problema fundamental en el proceso de generar explicaciones y conocimientos sobre fenómenos que se dan en el acontecer social. Expresa un problema epistemológico que se refiere tanto a la relación sujeto/objeto como a la construcción de lo concreto/abstracto, a la relación entre la reconstrucción empírica de la realidad y la teoría que debe resolverse en el ámbito del modo en que el sujeto la piensa. Fundamentalmente porque todo conocimiento brota del sujeto (Husserl 2005).

Por esta razón, los indicadores no representan únicamente la expresión morfológica del objeto (o sea, la forma de presentarse del fenómeno), así como tampoco representan la traducción empírica de la teoría, en 
el sentido de ser la operación de los conceptos o producto de la definición de categorías, dimensiones o variables. El problema es más complejo, dado que se refiere a la propia estructura sintética del objeto de estudio, al objeto como totalidad concreta.

Ciertamente, existen diversas concepciones y maneras de entender y definir el papel de los indicadores, así como su función en el proceso de investigación. Habría que hacer aquí una primera distinción relacionada con las perspectivas, usos y sentido que tienen los indicadores:

Perspectiva 1. Para ordenar y sistematizar información para la planeación, evaluación y toma de decisiones, donde se constituyen en sistemas de información que dan cuenta de conocimientos descriptivos sobre las características cuantitativas de un ámbito institucional, económico, geográfico, cultural, educativo, etcétera. Se presentan en censos, bancos de datos, entre otros referentes de contextos regionales, nacionales o supranacionales.

Perspectiva 2. Se construyen para recabar y sistematizar información para el análisis estadístico sobre problemas y fenómenos sociales. Es una traducción de los conceptos y nociones teóricas en unidades de análisis, como unidades de medida para cuantificar, de manera matemáticamente precisa, la realidad objeto de estudio.

Perspectiva 3. La construcción de indicadores como parte de una estructura sintética o totalidad concreta, es el modo morfológico de presentarse del objeto. La realidad se reconstruye yendo de lo concreto a lo abstracto y viceversa para establecer la expresión teórica y empírica del objeto.

La Perspectiva 1 tiene que ver con la necesidad de contar con índices (series de indicadores) para el ejercicio gubernamental, institucional o de la cuantificación de los aspectos más relevantes de las distintas esferas de las actividades que se generan en los sectores económico-sociales. Constituyen estadísticas y referentes básicos para tener una representación cuantitativa de la realidad social. La tendencia para construir sistemas de información cobró particular importancia a partir de la posguerra y se convirtió en una necesidad tanto de gobiernos locales, como de organizaciones supranacionales (ONU, UNESCO, OCDE, BM, entre otras).

Estos índices han tenido una utilidad diversa y diferenciada: principalmente son usadas como referentes cuantitativos para medir los grados de crecimiento de un país; también son usados con un sentido comparativo, para medir los avances y desniveles entre los países o regiones. Esto ha permitido tener una noción que permite identificar y comparar los principales rasgos económicos, políticos y sociales. En cualquier caso cada institución, incluso cada gobierno local propone, de acuerdo a sus propósitos, el tipo de indicadores y los procedimientos para construirlos.

La Perspectiva 2, está relacionada con ciertas tendencias dominantes de la investigación en ciencias sociales en donde "son especialmente importantes los conceptos empíricos, los que tienen referentes más o menos directos de su significado en la experiencia sensorial, son los que pueden referirse a cosas observables... la lógica de la relación entre los conceptos y sus indicadores empíricos, pues, es que los segundos representan a los primeros y su variación está ligada a éstos" (Pasquino 1996:49-53). En esta perspectiva el sujeto se superpone al objeto, de tal manera que la técnica y los procedimientos estadísticos son más importantes que el objeto. 
Los indicadores son datos cuantitativos, producto de los procedimientos establecidos por el investigador, quien genera resultados que todos pueden observar de la misma manera, pero son hallazgos del investigador, no del objeto; las metodologías y las técnicas de medición adquieren mayor relevancia que el conocimiento de la realidad. Resulta más atractivo para el investigador el interés por la medición precisa que la explicación y conocimiento del objeto.

La relación sujeto-objeto se presenta como una relación cómoda si se da por sentada la existencia del objeto, la teoría que lo explica y las estrategias metodológicas para verificar su modo morfológico de presentarse. Con la aplicación de fórmulas regulares e itinerarios predeterminados el conocimiento es predecible y sólo requiere ser confirmado.

La construcción de indicadores tiene que ver con la necesidad de medir empíricamente un fenómeno, ¿quién define lo que se debe medir?, ¿el investigador?, ¿las necesidades de explicación del objeto? De tal manera que los indicadores se consideran como: a) descripción de los atributos morfológicos del objeto, b) objetivos, en cuanto son cuantificables y c) unidades de medida para el análisis morfológico, basado en metodologías experimentales.

Así, es posible considerar la relación entre los conceptos como la representación empírica organizada en variables y dimensiones, siempre ligadas al concepto operativo.

Lo que se refiere a la determinación, por parte del investigador, de los contenidos empíricos de los conceptos.

De tal manera que la lógica de construcción de indicadores sería:

-Representación literaria. Construcción abstracta del problema, para establecer las regularidades y plantearlo como un problema de medida.

-Especificación del concepto. Aspectos o dimensiones deducidas analíticamente o por sus interrelaciones empíricas.

-Elección de indicadores. Definir los indicadores de los aspectos o dimensiones (Boudon y Lazarsfeld 1985:36).

La cuestión de la relación sujeto-objeto, en este caso, se resuelve por el qué piensa el sujeto más que cómo piensa, en función de la posibilidad operativa, de medida del objeto. En otras palabras, el sujeto da cuenta del fenómeno que estudia a través de indicadores y de acuerdo a variables, a elementos de variabilidad del fenómeno determinados por el investigador y a parámetros o dimensiones operativas construidas externamente al objeto.

La Perspectiva 3, relacionada con la expresión teórica y empírica del objeto, implica una ruptura con su modo morfológico de presentarse. Es decir, no es lo morfológico por sí mismo lo que determina la construcción de los indicadores, sino que es la reflexión del sujeto sobre los distintos planos y articulaciones del fenómeno, visto desde diferentes puntos de observación, en tanto el objeto tienen múltiples significados y que a pesar de constituir una realidad determinada, por el propio recorte que se plantea para pensarla, no parte de una preexistencia. 
Esto significa que la construcción de indicadores no responde a una conexión inmediata con la realidad a partir de una teoría preexistente, sino que responde a mediaciones articuladas por una teoría propia del objeto; conceptos pensados a partir del objeto y sus múltiples articulaciones en un momento en que éste está dado dentro de procesos heterogéneos. De lo que se trata, en esta perspectiva, es de ubicar las distintas facetas del fenómeno en su modo morfológico de presentarse, verlo desde diferentes puntos de observación para identificar lo determinado del fenómeno.

El sujeto reflexiona sobre los contenidos teóricos y empíricos del objeto a partir de su problematización, construyendo los indicadores para el análisis morfológico del fenómeno. Hay un modo de razonamiento que busca reconstruir la realidad en sus distintos contenidos empíricos desde distintas ópticas y formas de reflexión. Aquí el sujeto no sólo cuestiona la realidad sino que también, y de manera relevante, cuestiona cómo la piensa, cómo se apropia de ella y cómo la reconstruye teórica y empíricamente. Zemelman (VV.AA. 1994:8) resume este proceso de la siguiente manera:

I) Comenzar por observar la realidad en un plano morfológico.

II) Problematizar la observación morfológica en términos del razonamiento constructor de conocimientos y no solamente de razonamiento explicativo.

III) Reflejar la problematización en una representación conceptual que contenga una potencialidad transformadora y no solamente como estructura teórica...

IV) Cuidarse de que las relaciones asuman la forma y contenidos de una opción teórica (...) que sirva de base para formular proposiciones teóricas.

V) Comprender al concreto reconstruido como el producto que resulta de la construcción del reflejo, lo que implica saber relacionar el momento de la primera visión de la realidad con el de la reconstrucción racional.

VI) En este proceso de elaboración tiene lugar la transformación del nivel morfológico inicial, de ser uno puramente descriptivo en otro dinámico y articulado, de manera que, sobre esta base de conceptualización se pueda aprehender lo concreto...

VII) La totalidad es la forma de entendimiento adecuada a la exigencia epistemológica de transformar lo aparente en realidad; lo cual consiste en la transformación de los conceptos mediante lo que percibimos y relacionamos intuitivamente con la realidad, en construcciones mediatizadoras y en ángulos de observación antes que en contenidos teóricos.

Este planteamiento representa el itinerario posible para la construcción de indicadores. Dicho de manera resumida, el sujeto reflexiona sobre el objeto a la vez que reflexiona sobre la manera en que se aproxima a él para reconstruirlo teórica y empíricamente. El sujeto piensa el objeto y reflexiona sobre cómo lo piensa. Es decir se coloca frente a la realidad para determinar los contenidos teóricos y empíricos y no al revés: pararse frente a la realidad con una serie de contenidos preexistentes al pensamiento, incluso a la realidad que se busca conocer. El problema de la relación sujeto-objeto se resuelve bajo el cuestionamiento sobre cómo el sujeto construye al objeto, cómo lo piensa y lo reflexiona. Esta es la lógica que se desprende de esta perspectiva y que se constituye en la base para la construcción de indicadores. 
De las tres perspectivas planteadas, las dos primeras se refieren a la morfología del objeto y la tercera al modo morfológico de presentarse el fenómeno. Dicho de otra manera, para las dos primeras la prioridad es el qué piensa el sujeto sobre la realidad y en la tercera el cómo la piensa. Así, el sujeto se plantea sobre qué piensa del objeto y no en el otro sentido sobre cómo lo piensa. Representan dos formas de resolver la relación sujeto-objeto y dos formas distintas de plantear la construcción de indicadores. De un lado lo determinado, el dato tal cual se presenta; de otro lo determinado-indeterminado a partir de la historicidad de la realidad.

Tanto para una perspectiva como para la otra, la función y el uso de indicadores es importante, de hecho fundamental para el proceso de conocer. Es fundamental, también, para el tránsito de lo abstracto a lo concreto, en tanto el objeto contiene elementos teóricos y empíricos y los indicadores posibilitan la aplicación de metodologías cualitativas o cuantitativas que conducen a una nueva teorización sobre el objeto, como teoría renovada, como un nuevo conocimiento. Aquí radica lo medular de su función.

Los indicadores son, entonces, la representación empírica del objeto de estudio (o una definición empírica de éste) y tiene que ver con la necesidad de cuantificar o medir un fenómeno. Como datos cuantitativos, permiten el uso de metodologías pertinentes para los propósitos que se desprenden de la relación sujetoobjeto. De ahí una buena parte de su importancia. En lo que respecta al orden cualitativo, la construcción de indicadores representa la determinación de unidades de medición a partir de los contenidos teóricos del objeto; corresponde a la construcción de la estructura teórica y su traducción en indicadores de las cualidades del objeto. Se trata, principalmente, de establecer una relación entre lo teórico y la definición de las cualidades, expresadas empíricamente en indicadores para el estudio del fenómeno, mediante estrategias de investigación cualitativas y de acuerdo a las propias necesidades de explicación del objeto. Lo que quiere decir que aunque haya un repertorio de metodologías cualitativas o cuantitativas, no son preexistentes al objeto, sino que se convierten en estrategias de investigación y explicación para dar cuenta del mismo.

Existen procedimientos simples y otros muy elaborados, pero su función es muy clara: indicar atributos empíricos del objeto de estudio. Hay clasificación de indicadores económicos y sociales, diseñados para la planeación y para los sistemas de información; por otro lado hay los que se relacionan con la actividad científica, particularmente con la investigación social. Estos últimos son de mayor interés. Hay una diversidad enorme de clasificaciones y características: cuantitativos y cualitativos; objetivos y subjetivos; simples y complejos; absolutos y relativos; autónomos e independientes; descriptivos y analíticos; internos y externos, etcétera. El asunto es que cada investigador los denomina de manera arbitraria por la tradición científica a la que se adhiere. Nombrarlos de tal o cual manera es irrelevante y no arroja mayor claridad sobre su función.

Una cuestión destacada en relación a los indicadores en ciencias sociales, es que no existen procedimientos definidos para su construcción; lo que significa que cada objeto de estudio tiene una forma específica de presentarse en su expresión morfológica. Cada objeto propuesto supone sus propios indicadores. Esta situación lleva a lo que podríamos ubicar como otra distinción relacionada con una diversidad amplia de concepciones, definiciones y propuestas para su estructuración y uso. 
Por otra parte es claro que los indicadores deben ser precisos, consistentes con los conceptos, construidos a partir del objeto de estudio y clasificados de manera sistemática, dado que constituyen unidades de análisis. Estas son características de forma, pues como problema epistemológico se resuelven de otra manera.

\section{Reflexiones Epistemológicas sobre la Construcción de Indicadores}

El problema de fondo en la construcción de indicadores no tiene que ver con la teoría misma o con la operación de los conceptos. La reflexión no está en relación a lo que se conoce, al conjunto de teorías acumuladas que conforman el bagaje intelectual en general y, de manera particular, respecto al objeto de estudio. La cuestión fundamental es cómo se piensa, cómo se apropia y cómo se construye el objeto. Dado que no es una mera exterioridad requiere ser procesado desde la subjetividad del sujeto, no como teoría o expresión empírica, ni como indicadores preexistentes, sino de acuerdo a la lógica del propio fenómeno. Esto le da un sentido dinámico a la relación sujeto-objeto.

Si el sujeto propone, establece un conjunto de indicadores desde su particular punto de observación, desde la teoría que posee, no implica que correspondan al modo morfológico de presentarse del objeto; o sea, no significa que sean indicadores que reflejen empíricamente al objeto. Lo que supone es que son indicadores del propio sujeto como exterioridad de él mismo.

Dicho de otra forma, no es el sujeto y su subjetividad, al modo de Husserl, quien construye los indicadores empíricos del objeto, sino su externalidad que aparece como "objetiva", por el criterio de una morfología y de una teoría preexistente. El sujeto se coloca frente a la realidad y define lo que observa a partir de la teoría y los conceptos que forman parte de sus saberes. No observa el objeto como tal sino la teoría que propone para observarlo. Los indicadores, entonces, no son del objeto sino de la teoría que el sujeto propone de manera "objetiva".

La lógica del sujeto desde esta óptica no es, por tanto, la lógica del objeto. Los indicadores se constituyen en indicadores de lo que el sujeto quiere ver del objeto, lo que no necesariamente coincide con su estructura sintética (es decir, con el conjunto de articulaciones y conexiones que determinan al fenómeno para presentarse de una forma específica). El problema de fondo no es, pues, qué se piensa del objeto, sino cómo el sujeto piensa la realidad para conocerla, cómo elabora y reelabora la teoría para resignificarla y cómo reconstruye el objeto empíricamente.

El objeto tiene una doble lógica: una interna y otra externa. La interna corresponde al propio movimiento, a la propia dinámica que generan los procesos al interior del fenómeno; la externa, se refiere al contexto, a las conexiones con otros segmentos de la realidad, en la cual se inscribe y de la cual se hace un recorte para delimitarlo. Estas lógicas se refieren a un tiempo propio y específico del suceder del objeto, dentro de un tiempo social, caracterizado por diversas temporalidades que suceden simultáneamente en la realidad como ámbitos temporales y espaciales de problemas posibles.

Por tanto, la lógica, (la doble lógica) del objeto no corresponde ni al tiempo ni al espacio del sujeto. Este debe reflexionarlo, considerar esas cualidades y su modo morfológico de presentarse. Entonces, el sujeto debe reflexionar desde la lógica interna y externa. El objeto es el resultado de un conjunto de cuestionamientos que permiten articular las formas de apropiación de la realidad y plantear las estrategias para conocerlo. 
El objeto se construye como estructura sintética o como totalidad concreta dado que contiene un conjunto de articulaciones e interacciones y múltiples determinaciones, tanto internas como externas, a partir de lo cual es posible su construcción conceptual y al mismo tiempo reconstruirlo empíricamente, lo que abre la posibilidad de construir los indicadores que reflejen la morfología del objeto.

De tal manera que lo fundamental, tanto para la reflexión teórica como para la definición de indicadores, es la construcción de la estructura sintética o la totalidad concreta del fenómeno. Ambas nociones dan cuenta del mismo proceso de reflexión sobre la realidad. Básicamente representan un modo de razonamiento.

La estructura sintética expresada en el tiempo y el espacio, supone una intencionalidad reflexiva del sujeto que define como problema de conocimiento un fenómeno, mediante un recorte temporal del espacio en el cual el objeto transcurre y define su síntesis para hacerlo inteligible. La relación entre el sujeto y el objeto representa una unidad en la que se sintetiza la temporalidad y la espacialidad de ambos. El objeto desde la perspectiva del sujeto y cómo éste lo percibe en sus distintas facetas temporales, es un fluir permanente del mismo objeto dónde el sujeto está implicado, ya sea desde el punto de vista reflexivo, desde la intencionalidad de conocer, o bien, desde la propia temporalidad del sujeto. Por tanto, no es un acto arbitrario, por el contrario, es un acto intencional para determinar la unidad, la conciencia de la unidad de las diversas variantes y facetas del objeto. El objeto y quien lo piensa se expresan en una unidad.

De acuerdo a lo anterior, construir el objeto consiste en determinar la estructura sintética del fenómeno que pensamos, es el primer paso para conocer el objeto en un mundo que se mueve velozmente, que cambia incesantemente. En términos de Kosik (1967), la definición de la estructura sintética correspondería a la construcción de la totalidad concreta, entendida como la expresión de múltiples determinaciones. No obstante, la totalidad y el todo no significan de ningún modo lo mismo. El todo es inasible como tal, es ininteligible. La totalidad se construye, el sujeto se apropia y la presenta como la síntesis de diversas articulaciones y facetas del fenómeno que se estudia. Zemelman (VV.AA. 1994:8) señala que "la totalidad es la forma adecuada a la exigencia epistemológica de transformar lo aparente en realidad, lo cual consiste en la transformación de los conceptos mediante lo que percibimos y relacionamos intuitivamente con la realidad, en construcciones mediatizadoras y en ángulos de observación antes que contenidos teóricos". Es decir, los indicadores que dan cuenta de los contenidos empíricos forman parte de la estructura sintética del objeto definida por el sujeto, lo que en Bachelard (1999) correspondería a la geometrización. En términos epistemológicos, representa el proceso mediante el cual se reflexiona sobre los contenidos abstractos y concretos, en un ir y venir de ambos planos de la realidad. Lo abstracto como la reflexión sobre la construcción teórica del objeto y lo concreto como la apropiación del modo morfológico de presentarse.

Esta reflexión abre amplias posibilidades de conocimiento, dado que no existe una teoría preexistente al objeto, tampoco existe su reconstrucción morfológica. El objeto es resultado de la aprehensión de la realidad, el producto de un ir de lo concreto a lo abstracto y nuevamente a lo concreto, para apropiarse y plantear una síntesis de lo dado a partir de problematizar la realidad y reconstruirlo teórica y empíricamente.

El proceso de reflexión involucra lo abstracto del objeto y sus formas de concreción. De tal manera que el sujeto construye la teoría a partir de esa conexión al interior del objeto. Es decir, el sujeto busca conocer lo que no conoce, por esa razón debe proceder a construir la estructura sintética el objeto, de tal modo que esté en condiciones de establecer las diferentes conexiones y articulaciones, reflexionar la realidad desde distintos puntos de observación. El proceso no es mecánico, en donde se agote primero lo concreto y luego 
lo abstracto, sino que es un cuestionamiento permanente entre cómo, a partir de qué, desde dónde y para qué se piensa el objeto para conocer su expresión empírica de manera teórica.

Los indicadores constituyen una definición empírica del objeto y son producto de una construcción teórica del sujeto sobre el fenómeno para descubrir lo específico concreto del objeto. Esto representa una primera parte del proceso de reflexión; de tal manera que los indicadores adquieren un sentido y una función de conocimiento, además de su utilidad como elementos para la medición y cuantificación. Nuevamente, el sentido de lo concreto no es solamente su forma de presentarse y ser explicado empíricamente, sino también y de manera relevante, la posibilidad de conocerlo a partir de su concreción, de las conexiones, articulaciones y determinaciones que hacen que el fenómeno se presente de una manera y no de otra, lo que le aporta un sentido lógico con lo teórico-abstracto.

Los indicadores son sólo útiles epistemológica y metodológicamente para determinar las características empíricas de lo dado, no para conocer lo dándose. De esta forma, el recorte que se hace de la realidad para delimitarla, parte de lo concreto, de la presentación empírica del fenómeno. Generalmente, este es el proceso a partir del cual el sujeto inicia la reflexión sobre el fenómeno para construirlo en sus diversas articulaciones hasta apropiarse de él como estructura sintética.

El objeto no existe como tal hasta que el sujeto lo problematiza, lo construye; por ello no existe una teoría que lo explique. Hay, sí, una acumulación de teorías y conceptos, un bagaje intelectual, pero nada que permita conocer al objeto de manera anticipada (carecería de sentido plantearse una tarea semejante). Se habla de un sujeto que busca conocer lo que ya conoce, utilizando metodologías orientadas a la medición precisa de lo ya conocido. En este caso, se puede hablar de un proceso concreto-abstracto entre una teoría preexistente y la mera cuantificación del fenómeno.

Por tales razones se hace necesario reflexionar sobre el objeto a partir de las necesidades de ser conocido y rescatar de los conceptos el potencial para nombrar lo nuevo del fenómeno. Se trata de repensar la teoría a partir del objeto y no de pensar el objeto a partir de teorías. Este es un aspecto importante de la relación concreto-abstracto-concreto, que se expresa en la construcción de indicadores.

Es correcto pensar que los indicadores se construyen a partir de lo concreto de los fenómenos, de los contenidos empíricos del objeto. Sin embargo, es necesario apuntar que lo concreto no es únicamente lo tangible, lo medible y lo cuantificable, sino que los fenómenos tienen diversas maneras o niveles de concreción, dentro de las cuales el modo morfológico de presentarse es una de ellas en su expresión más inmediata. El tiempo y el espacio también son maneras de concretarse de los fenómenos dentro de los recortes posibles de la realidad; de la misma forma, las situaciones históricas y el mismo carácter de historicidad del objeto y del sujeto, en tanto interacciones de diferentes planos de la realidad. Por estas circunstancias, los indicadores son producto de la relación abstracto-concreto. Los indicadores se derivan de la teoría construida a partir del objeto, con un contenido teórico conceptual propio y empíricamente pertinente.

A su vez, los indicadores representan el punto de articulación, tanto para el conocimiento empírico como para iniciar una nueva fase concreto-abstracto, en el sentido de teorizar o nombrar conceptualmente al objeto, generar un conocimiento novedoso sobre un segmento de la realidad, dar cuenta teóricamente del objeto, a la vez son la base para la definición y el uso de metodologías de forma pertinente para medir o cuantificar al objeto. 
Queda claro que la construcción de indicadores no es una cuestión arbitraria en manos del sujeto y a pesar de que no existen procedimientos para tal propósito (pues no es necesario que existan), éstos se definen como resultado de la reflexión del sujeto que piensa teóricamente al objeto, sobre el modo morfológico de presentarse de éste.

Cada objeto tiene sus propios contenidos teóricos y empíricos, su propia exigencia de razonamiento y, desde luego, su propia exigencia para la construcción de indicadores. Queda claro, también, el papel de los indicadores en el proceso de construcción de conocimiento y el fondo epistemológico para resolver el problema de su definición.

Sin embargo, no es fácil asumir este compromiso epistemológico, como tampoco es fácil entender y aceptar estas exigencias al pensamiento. Hace falta la voluntad, la intención de conocer del sujeto, en tanto que todo conocimiento emana de éste. Dice Bachelard (1988:9) que "la razón conoce sólo lo que ha logrado aprender. Y sin embargo para pensar, en primer lugar habría tantas cosas que desaprender". Lo que significa que el sujeto tiene que asumir el compromiso de desaprender una cultura científica que plantea la voluntad del sujeto como prevaleciente sobre el objeto. Dicho de otra manera, una cultura científica en donde se busca conocer lo ya conocido con métodos y teorías preexistentes al objeto; agrega: "por lo tanto, es necesario que el hombre de ciencia reaccione contra el pasado de su propia cultura. Se debe practicar una especie de psicoanálisis, para impedir que el espíritu se anquilose en sus propias ideas claras" (1988:10).

Se estableció que la cuestión de fondo de los indicadores constituye un problema epistemológico. Husserl (2005) señala que el sujeto es la fuente de todo conocimiento y que se trasciende en el objeto: es decir, la subjetividad del sujeto se objetiva en el objeto, pero sólo trasciende cuando construye una estructura sintética, construye el objeto y se transforma en un verdadero sujeto que conoce.

Bachelard plantea que se trata de que el sujeto refunde sus formas de pensar el objeto. Refundar la intencionalidad y los horizontes de conocimiento. Donde el sujeto debe formarse reformándose.

En ambos autores la propuesta es abrir los horizontes del conocimiento y la voluntad de conocer. Lo que implica una ruptura con las formas de cómo, qué, para qué y por qué pensamos la realidad. Así, en el proceso de conocer, la ruptura es la ruptura del propio sujeto a través de una doble relación crítica con el objeto, de ruptura y reorganización del pensamiento.

Doble relación permanente que se refiere a la intencionalidad, a la conciencia del sujeto como constructor de conocimiento. Ruptura con las aproximaciones fundadas en propuestas meramente experimentales; vigilancia sobre el uso de recursos teóricos e instrumentales, herramientas empíricas preexistentes y la consecuente reorganización de las formas de apropiarse del objeto de manera vigilante en la construcción teórica y empírica, de acuerdo a lo que el objeto requiere para ser conocido.

Este proceso significa una ruptura epistemológica que requiere voluntad y compromiso; el sujeto debe, entonces, romper con la pretendida preexistencia del método, igual que debe romper con la teoría como un marco conceptual que todo lo explica y con el uso de metodologías estandarizadas y, desde luego, romper con una cultura científica cerrada y estática.

Bourdieu (2003) sugiere una doble ruptura. Propone no sólo asumir la crítica epistemológica de la cultura científica, de los métodos, teorías, instrumentos y herramientas metodológicas, sino también asumir la 
crítica de las condiciones en las que se desarrolla el trabajo científico, la propia estructura organizativa de la ciencia, que tienen una función muy importante en la orientación, prácticas, resultados y usos de la investigación. Es decir, asumir la crítica sobre las múltiples determinaciones de esa estructura de poder sobre el trabajo científico.

Una doble ruptura epistemológica que supone cuestionar las propias implicaciones del método, la teoría, las metodologías y las condiciones institucionales en las que se desarrolla el trabajo científico. Se refiere Bourdieu a las condiciones que se han generado en la construcción del campo científico: Estructuras académicas, financiamientos, espacios de poder que dan lugar a tensiones y confrontaciones que los investigadores padecen o disfrutan, mediadas por intereses de grupo hasta conformarse en un habitus, que involucra la relación tensión-alianza-competencia entre investigadores, hasta la adquisición de estructuras teóricas y metodologías, de acuerdo a las corrientes científicas dominantes.

En ese contexto, se entiende que ya no es suficiente reflexionar únicamente sobre los métodos y las teorías y sus implicaciones en la producción de conocimiento, sino también en las condiciones en que éste se genera como un campo con características propias, en donde las relaciones entre científicos se constituyen en un mundo social, con su correspondiente impacto sobre cómo se produce el conocimiento y en la noción misma de realidad. En tanto las condiciones externas determinan las prácticas y los resultados del trabajo científico, suponen ya un problema epistemológico: por ello es necesario reflexionar en esa doble ruptura.

La necesidad de repensar sobre las estructuras organizativas de la ciencia y sobre las instituciones académicas, ya había sido señalada de manera puntual por C. Wright Mills cuando se refiere al "ethos burocrático" (1997:116). La idea de campo y habitus representa un impulso renovado para repensar sobre esas condiciones. Bourdieu (2003: 14-15) lo expresa de la siguiente manera: "Bachelard observaba que la epistemología había reflexionado en exceso sobre las verdades de la ciencia y no suficientemente sobre sus errores de la ciencia en trance de construcción, sobre el proceso científico en sí mismo...la visión realista y, a menudo, desencantada que se ha formado de las realidades del mundo científico los ha llevado a proponer una teoría relativista, por no decir nihilista, que marcha a contracorriente de la representación oficial de la ciencia. Ahora bien esta conclusión no tiene nada de fatal y es posible, en mi opinión, asociar una visión realista del mundo con una teoría realista del conocimiento. Y ello a condición de operar una doble ruptura con los dos términos del binomio epistemológico formado por el dogmatismo logicista y el relativismo que parece inscrito en la crítica histórica... cierto número de investigaciones, que asimilan el mundo científico al mundo artístico tienden a reducir la actividad del laboratorio a una actividad semiológica: se trabaja sobre unas inscripciones, se hace circular unos textos... ¿es un campo como los demás? Y en caso contrario ¿cuáles son los mecanismos que crean su especificidad y, simultáneamente, su irreductibilidad a la historia de lo que allá se engendra?"

Lo que sugiere Bourdieu es justamente una ruptura con los determinantes internos de cómo se genera conocimiento y con los mecanismos externos a partir de los cuales se organiza la actividad científica. Todo ello involucra tanto orientaciones teóricas, metodológicas e instrumentales, como aspectos organizativos, financieros, espaciales, políticos, etcétera, a partir de lo cual se construye un campo y un habitus científico. En eso consistiría la doble ruptura epistemológica.

La propia idea de realidad se pone en juego, los puntos de observación, la teoría y la metodología y desde luego no sólo la construcción de indicadores, sino su uso y su papel en la producción de conocimiento. 
Por estas razones señalamos que el fondo en la construcción de indicadores se resolvía de otra manera, y, en efecto se resuelve a través de la reflexión epistemológica. Como señala Zemelman (1989) representan la punta del iceberg, tanto como problema del conocimiento como de las condiciones en que éste se genera; de una problemática teórica-empírica, abstracto-concreta, en donde el objeto es el punto de articulación de la teoría, de los contenidos empíricos y sus indicadores, que es posible reconstruir a partir de cómo piensa el sujeto la realidad, cómo construye el objeto a partir del recorte de la realidad para apropiarse de lo dado y con qué exigencias de pensamiento el sujeto reconstruye lo concreto y lo abstracto. En síntesis, la construcción de los indicadores representa un problema epistemológico y metodológico a la vez.

\section{El Objeto de Estudio y la Construcción de Indicadores}

Una vez que el sujeto aprehende la realidad dada, se apropia de ella y la reconstruye con una serie de significados pertinentes (lo pertinente está en función de la teoría que se construye a partir del modo morfológico de presentarse del objeto), se halla en una condición de reconstruirla empíricamente, es decir, de definirla en términos de indicadores, de unidades de análisis. Los indicadores expresan una síntesis de lo abstracto-concreto, una síntesis del pensamiento teórico y la definición empírica del objeto; lo que representa la forma epistemológica en la cual el sujeto resuelve su relación con el objeto. “De esta manera, los objetos de la ciencia en vez de ser pobres abstracciones extraídas de la riqueza de lo concreto, son productos regulados teóricamente y ordenados materialmente de un trabajo que los dota de la riqueza de las determinaciones del concepto y de toda la sensibilidad de las precisiones experimentales. Entonces se puede decir, con Bachelard, que esos objetos son abstracto-concretos" (Lecourt 1978:34).

De tal modo que los indicadores no son una expresión ni un reflejo mecánico de los conceptos reconstruidos, resignificados de la realidad dada.

Tampoco existe una arbitrariedad en su definición. Son producto, los indicadores, de la construcción de la estructura sintética (del modo abstracto-concreto de darse de los fenómenos) del objeto, de la manera que el sujeto resuelve su relación con la realidad. Así como el sujeto entabla una relación de ir y venir frente a lo concreto-abstracto del objeto, pasa de una postura epistemológica a una metodológica y viceversa, tanto para construir los conceptos pertinentes como sus indicadores.

En este proceso no existe una teoría, ni indicadores preexistentes; el sujeto se enfrenta a lo indeterminado de una realidad que se encuentra en un proceso incesante de lo dado-dándose. La apropiación-construcción de lo dado significa la conquista de lo indeterminado para convertirlo en un fenómeno determinado teórica y empíricamente, que a su vez se abre a lo indeterminado.

Por tanto, no existe un objeto determinado, en el sentido de preexistencia, sino que es un producto de un proceso de aprehensión-apropiación de una realidad dada por parte del sujeto, un esfuerzo por conquistar y construir el objeto, conocerlo y dar lugar, de nueva cuenta, a lo indeterminado, a lo dándose de la realidad en diferentes planos y temporalidades y su expresión en una multitud inmensa de fenómenos.

Por tal razón, la construcción de indicadores no es solamente un problema de operar los conceptos para elaborar índices, definir dimensiones y variables contenidos en los propios conceptos, sino que: "La cuestión de fondo es poder situar a la teoría en lo real antes que a lo real en la teoría; por ello, antes de teorizar derivado de proposiciones desde un marco teórico, hay que reconstruir la situación de acuerdo a una lógica abierta a la objetividad y aprehender lo real desde ella... de este modo se establece la diferencia entre la 
jerarquía definida por exigencias teóricas y la jerarquía posible de establecerse por exigencia de lo real sobre lo teórico: el rompimiento de lo determinado por lo indeterminado" (Zemelman 1989:164).

En esta perspectiva habrá que ver lo determinado como lo dado en el contexto de lo dándose, es decir, de lo indeterminado. Lo determinado es producto de una construcción, de una apropiación de lo real para construirlo como una estructura sintética con contenidos teóricos y empíricos. Pero esto sólo puede ser posible con la voluntad o intensión de conocer a partir de rupturas del pensamiento, de cómo piensa el sujeto la realidad, y la consecuente reorganización de sus reflexiones.

El sujeto progresa en sus reflexiones a partir de sus propias formas de reorganizar su pensamiento, de las sucesivas rupturas frente y desde el objeto en un proceso de apropiación para conocerlo. De tal manera que el sujeto en sus rupturas se confronta con su propio pensamiento de manera continua para construir la estructura sintética del fenómeno. Rupturas-reorganización habrá que verlas en la perspectiva anteriormente expuesta de la doble ruptura epistemológica.

De tal manera que esos procesos de ruptura-reorganización del pensamiento frente a lo determinadoindeterminado, pone al sujeto en condiciones de dar cuenta empíricamente del objeto a través de la construcción de indicadores. Se ha visto que éstos representan un punto fundamental de la articulación entre lo concreto y lo abstracto; son fundamentales para definir las metodologías pertinentes, tanto desde el punto de vista cuantitativo como cualitativo.

En uno o en otro caso, los indicadores permiten mediciones matemáticamente precisas o análisis cualitativos, en tanto que lo que se mide o analiza, el dato o la opinión, tendencia, actitudes, etcétera, se plantea a partir de los indicadores. En otras palabras, lo que se cuantifica y analiza son los indicadores. El uso de instrumentos y herramientas metodológicas es para buscar, medir y encontrar estos rasgos en el objeto, en la realidad empírica. Los indicadores le aportan orden al proceso de producir información y datos. Es decir, la realidad empírica se reconstruye a partir de los indicadores. Entonces hay una conexión epistemológica y metodológica entre lo abstracto-concreto, teórico-empírico al momento de reconstruir la realidad en su modo morfológico de presentarse. Esta es otra razón para destacar la importancia de los indicadores en el proceso de construir conocimiento.

No hay, o no debería haber, metodologías preexistentes al objeto, a sus propios indicadores, no puede haber si no se sabe que se va a medir (salvo que se busque conocer lo ya conocido y se utilice metodologías para la medición precisa de lo ya conocido). Lo que hay son instrumentos y herramientas metodológicas tanto de corte cualitativo como cuantitativo. Es el mismo problema que se plantea con una teoría preexistente, lo que se llama marco teórico, que da cuenta de objetos ya conocidos; en donde no hay rupturas ni reorganización del pensamiento; no es el cómo sino el qué piensa el sujeto lo que importa. Esto significa, por otro lado, que las metodologías se desprenden, se estructuran o construyen a partir de los contenidos teóricos y empíricos, de la estructura sintética del objeto. Dicho de otra manera, la forma en que el sujeto resuelve la relación abstracto-concreto.

Por otra parte los indicadores representan una doble función. De un lado, articulan lo abstracto-concreto, lo determinado y lo dado del objeto, el recorte de la realidad expresado como estructura sintética. De otro, representan la apertura a lo indeterminado, a lo dándose, a la posibilidad de nuevas teorizaciones sobre y a partir de la información, datos y elementos empíricos producto de la aplicación de metodologías; es decir, teorizar a partir de los resultados de la medición, el análisis y sistematización de los indicadores. Esto es, 
pasar de lo abstracto a lo concreto y elevarse a lo abstracto, produciendo nuevos conceptos, resignificándolos.

La producción de nuevos conceptos o de la teoría resignificada (al modo propuesto por Zemelman) depende no sólo de los datos, sino de la reflexión que se hace de los resultados producto de la medición de los indicadores, y esto, al menos por los siguientes motivos:

1) Los indicadores representan la reconstrucción empírica del objeto. La descripción del modo morfológico de presentarse del objeto.

2) Son el punto de articulación de lo abstracto-concreto, lo teórico y lo empírico del objeto.

3) Son elementos a través de los cuales puede resolverse la relación epistemológica entre el sujeto y el objeto.

4) Articulan y permiten definir el uso de metodologías pertinentes, de acuerdo a las necesidades de conocimiento del objeto.

5) Permiten ordenar la reflexión para teorizar sobre el objeto a partir de los resultados de la aplicación de metodologías cualitativas o cuantitativas.

6) Abren la posibilidad a lo indeterminado desde lo determinado del objeto.

De acuerdo a lo hasta aquí expuesto se puede apreciar que la cuestión de los indicadores no constituye un problema menor, sino por el contrario, su centralidad "permite comprender en qué sentido hay que entender el término 'producción'; no sólo producción 'teórica' de conceptos, sino indisociablemente producción material del objeto de trabajo teórico, de lo que ya no se puede denominar 'dato' sino más bien su materia" (Lecourt 1978:33). Necesariamente habrá que ver, entonces, a los indicadores y su construcción desde una lógica de ruptura-reorganización del pensamiento del sujeto.

\section{Un Modo de Construcción de Indicadores}

Establecer metodologías previamente a la construcción de la estructura sintética del objeto, representa que, para el sujeto, la realidad se encuentra determinada y no es, por tanto, producto de un proceso de apropiación, de conquista y de construcción de lo determinado, de lo dado en el movimiento incesante de una realidad que se encuentra dándose, una realidad indeterminada.

Desde esta óptica, el sujeto recorre, por decirlo de alguna manera, el proceso de construcción del objeto de manera parcial. Lo inicia desde el punto de la existencia misma del objeto, lo ya dado, lo ya determinado, y a partir de ahí define los indicadores y las metodologías, también como algo ya dado para dar cuenta del fenómeno que eligió para medir. De antemano el sujeto sabe que tiene la necesidad de medir empíricamente el objeto y que para ello existe la caja de herramientas (al modo de Elster 2000), los instrumentos, técnicas y metodologías necesarias para tal propósito. De modo que no existen diferencias entre los objetos a medir; es, entonces, un problema de elección del sujeto, al margen de las necesidades explicativas del objeto. "En el lenguaje de las ciencias sociales son especialmente importantes los conceptos empíricos, los que tienen referentes más o menos directos de su significado en su experiencia sensorial; son las que pueden referirse a los observables. Dado que los significados en tales conceptos tienen en nuestra 
mente la forma de palabras y, a su vez, se refieren a un conjunto de objetos, de referentes... Para hacer frente al problema de la formación de conceptos empíricos, una primera regla de oro es la de recurrir explícitamente a definiciones verbales (o declarativas), que eliminan la ambigüedad del término, allanando de entre los distintos significados posibles el seleccionado previamente y que se mantendrá a lo largo de la investigación" (Pasquino 1996:49).

La cita es muy clara para ilustrar que existen diferentes formas de aproximarse a la realidad para conocerla. No es la idea hacer una crítica de éstas, sino establecer los alcances e implicaciones. Es decir, las lógicas que le dan diferentes sentidos a la relación abstracto-concreto, sujeto-objeto. Poner en el centro de la reflexión el cómo se piensa la realidad, desde dónde, desde qué puntos de observación el sujeto se sitúa para saber lo que sabe de la realidad (la teoría preexistente de un objeto igualmente determinado), para definir cómo medirlo.

Lo fundamental es el sujeto y cómo piensa la realidad. Cómo la explica; no tanto lo que sabe, ni cuánta teoría acumulada posee acerca del objeto, sino su capacidad para cuestionar ese saber, su capacidad para resignificar los conceptos en función del objeto de estudio (Zemelman 2005:63), dado que todo conocimiento emana del sujeto entendido como sujeto social. Lo verdaderamente importante es la ruptura epistemológica, una crítica al pensamiento para reformarlo, reorganizarlo.

Por estas razones, la función de la teoría va más allá de la mera descripción declarativa, como se señala en la cita, para buscar estudiar la teoría relativa al problema, lo que los autores dicen o han dicho sobre el fenómeno para delimitar los propios contenidos teóricos del objeto. Resignificar la teoría en función del objeto de estudio para dar cuenta de realidades concretas y no para inventar realidades. En otras palabras, de lo que se trata es de pensar la teoría para preguntarse cómo ésta puede, si lo puede hacer, nombrar a la realidad.

Esta cuestión es importante, pues la construcción de indicadores depende de este proceso, ya sea en la orientación de una descripción verbal (con un limitado contenido abstracto) de un objeto predeterminado por el interés del sujeto; o bien, repensar la teoría críticamente en función de su capacidad para explicar el objeto y en esta medida replantearla. Ruptura necesaria en tanto que se reflexiona sobre lo determinadoindeterminado, lo abstracto-concreto del objeto.

Entonces, los indicadores tienen un sentido más explicativo que descriptivo y las metodologías, técnicas, herramientas e instrumentos estarán definidos por los contenidos teóricos del objeto, con lo cual se resignifica también el sentido de la medición. El contenido empírico del objeto será correspondiente y resultado de esta ruptura y reorganización del pensamiento. Es decir, lo real se construye a partir de una serie de rupturas del pensamiento, tanto en sus contenidos teóricos como empíricos, lo que conduce al uso pertinente de metodologías, ya sean de orden cualitativo o cuantitativo. La utilización de estas metodologías es, entonces, un producto de esas rupturas y reorganizaciones del pensamiento para hacer inteligible la realidad y no el inicio de la investigación.

Por otra parte, en este proceso de construir conocimiento existen otros problemas que es preciso reflexionar sobre sus implicaciones. El primero de ello es la idea de realidad; el segundo, tiene que ver con la distinción entre tema, problema y objeto. Son dos aspectos que acercan al sujeto a la condición de construir indicadores, definir metodologías pertinentes, al uso de herramientas e instrumentos, a la posibilidad de 
teorizar sobre el objeto en una relación abstracto-concreto-abstracto y a la apertura a lo determinadoindeterminado.

La realidad es inasible como tal, está compuesta por un conjunto indeterminado de procesos, de tiempos, heterogénea en sus modos morfológicos de presentarse y con potencialidades diversas. Como tal, entonces, es ininteligible, incognoscible más allá de su manejo funcional en la vida cotidiana; llena de sueños, de fantasías y prejuicios bajo el predominio de la inmediatez y el conocimiento de las esferas de la experiencia diaria, a partir del sentido común. En este escenario no hay sujetos, hay individuos que construyen su pensamiento a través de la experiencia sensible en un mundo donde no hay exigencias conceptuales. Es una realidad con significados prácticos e instrumentales.

De tal manera que el individuo elabora conceptos, ideas que dan cuenta, que definen, explican operativamente el mundo. Teoriza, aunque su manera sea superficial, sin desarrollo, sin rigor. No está obligado a otra cosa, no hay mayor exigencia y no tiene por qué haberla. Operar en ese nivel es suficiente para la existencia diaria y su devenir, no es necesario nada más. Para este individuo lo real y la realidad coinciden. No existe la necesidad de razonamiento o de teorización, no existen problemas epistemológicos, ni metodológicos.

Sin embargo, pensar en el contexto del sentido común en algún aspecto de la realidad tiene un contenido orgánico, como lo delimita Gramsci (Sacristán 1998), un buen sentido, un núcleo sano, un potencial de conocimiento que el individuo sólo puede mirar si tiene la voluntad de saber, la intención de conocer. En el momento en que se asume esa posibilidad, dadas las condiciones, circunstancias y exigencias, el individuo se convierte en sujeto productor de conocimiento para cuestionar, problematizar y reflexionar sobre los fenómenos y cómo los piensa. Se pregunta qué es lo que no ve más allá del modo morfológico de presentarse el fenómeno.

Teorizar es distinto, es una exigencia de un razonamiento de mayor refinamiento conceptual. La realidad a través del cuestionamiento se presenta ya no como el todo, sino cómo una totalidad concreta; un recorte en el devenir del fenómeno para establecer lo dado, lo determinado del fenómeno y construir su estructura sintética.

Desde esta óptica, mientras que el individuo se apropia del mundo a través de conceptos de uso cotidiano, que le son funcionales, opera en un nivel igualmente funcional de abstracción.

El sujeto cuestiona y problematiza para construir una realidad dada, en donde ya se supone un nivel de abstracción más profundo, exigente y de una mayor elaboración teórica. Digamos que la distinción se da a partir de cómo y para qué se piensa la realidad. El primer nivel de pensamiento es operativo y no trasciende la estructura de la vida cotidiana, la zona de operación de la acción directa del individuo (Schutz y Luckmann 1973), constituye una apropiación funcional de la realidad. En el segundo caso el sujeto construye la realidad y la trasciende al generar nuevos conocimientos; lo que constituye una exigencia de pensamiento y de abstracción no solamente de mayor profundidad, sino también de mayor refinamiento. 
Todo esto es importante de señalar pues es preciso dejar bien claro que en el proceso de conocer existen diversas aproximaciones a los fenómenos, que generan diferentes niveles de abstracción y teorización a partir de exigencias epistemológicas y metodológicas diferenciadas.

Ahora bien, otro problema de orden epistemológico que es necesario mencionar y en el que se inscribe la construcción de indicadores, es el relativo a lo que constituye el tema, el problema y el objeto. Al respecto se genera mucha confusión en la construcción de la estructura sintética del objeto de estudio, al no distinguir lo que cada uno significa en ese proceso. El tema representa un enunciado general sobre el fenómeno desde su propia morfología, incluso desde la propia teoría relacionada con el fenómeno o podríamos decir que representa una primera definición, una primera delimitación teórica o empírica en el proceso de construcción de la realidad, en el sentido descrito anteriormente. Es un proceso inicial del sujeto que se plantea conocer el fenómeno más allá de las formas y recetas impuestas por la estructura de la vida cotidiana, esta exigencia constituye un esfuerzo epistemológico, que tiene que ver con el qué piensa el sujeto.

La segunda cuestión es sobre el problema, ya no es en este caso el sentido de lo que se piensa, si no la capacidad del sujeto para cuestionar esa primera delimitación del fenómeno; para preguntarle a la realidad por qué es que se presenta como tal; cuando el sujeto se pregunta qué es lo que no ve de lo que está viendo. Es decir, cuestionar sus propios modos de observar tanto como desde dónde observa y sobre todo cómo está pensando lo que observa; en síntesis, problematizar la realidad. Lo que significa "estar dispuestos a zambullirse debajo del nivel del agua y comenzar a ver que hay más allá de la superficie... si queremos zambullirnos para ver lo que hay debajo del iceberg, debemos tener la capacidad crítica, y la capacidad crítica significa no contentarse con lo que se ve, con lo observable" (Sánchez y Sosa 2004:28-29). Este segundo aspecto representa un problema de índole epistemológico, pues supone la doble ruptura: primero con el cómo pensamos y segundo desde dónde pensamos, lo que conlleva a la reorganización del pensamiento.

De hecho el sujeto está en una ruptura-reorganización del pensamiento de manera constante. Lo que significa que en el proceso de problematizar la realidad no hay una única ruptura, sino diversas de acuerdo a la capacidad del sujeto de cuestionar, problematizar y preguntarse permanentemente sobre lo que no ve, pues lo que ya ve es de por-sí evidente o ya lo ha expresado objetivamente. Lo cual es necesario para construir los indicadores o apropiarse empíricamente del objeto.

El problema no constituye el objeto. Esta tercera cuestión tiene que ver con la construcción de los puntos de articulación, entre lo dado y lo dándose, lo indeterminado y lo determinado, entre la interacción de los elementos teóricos y empíricos que se identifican a partir de la problematización y se articulan en una estructura sintética o totalidad concreta, expresada en el tiempo y el espacio, que supone una intencionalidad reflexiva del sujeto, que define como problema de conocimiento un fenómeno mediante un recorte temporal del espacio, en el que el objeto se construye y se define su síntesis para hacerlo inteligible.

La relación entre el sujeto y el objeto representa una unidad en la cual se sintetiza la temporalidad y la espacialidad de ambos: el objeto desde el punto de observación del sujeto y cómo se percibe el objeto en sus distintas facetas temporales en un fluir permanente del mismo objeto y en donde el sujeto está implicado, ya sea desde un punto de vista reflexivo, desde la intencionalidad de conocer, o bien desde la propia temporalidad del sujeto. Por tanto no es un acto arbitrario sino, por el contrario, un acto intencional 
para determinar la unidad de las diversas variantes y facetas del objeto en el fluir de su temporalidad; el objeto y quien lo piensa se expresan en una unidad sintética.

La estructura sintética se construye a partir de problematizar la realidad, de construirla en sus contenidos teóricos y empíricos. Ya no la teoría o las formas empíricas preexistentes sino la teorización y la reconstrucción empírica desde las necesidades de conocer el objeto, o sea la condición de poder determinar lo abstracto-concreto-abstracto del objeto, es decir, pasar de lo teórico a lo empírico (la reconstrucción a través de indicadores) y nuevamente a teorizar para dar cuenta del objeto.

En este marco, la teoría juega un papel central pero siempre desde el punto de vista de resignificarla para nombrar las realidades nuevas; extraer el potencial explicativo de los conceptos desde las necesidades de explicación del objeto o construir conceptos nuevos. Veamos una forma de plantear la construcción del objeto, expresado cómo estructura sintética. Pongamos un caso para ilustrar como plantear la construcción de los indicadores.

\section{A Modo de Conclusión: Un itinerario para la construcción de indicadores}

Como un ejemplo, y sin que esto signifique una revisión exhaustiva, se puede proponer como tema general "La percepción de la exclusión social en la periferia de una ciudad media en América Latina". Es un tema sugerente, cuya relevancia radica en: 1) la vigencia de la discusión sobre la exclusión social en el marco de los procesos de globalización y 2) el crecimiento demográfico y la expansión urbana en una ciudad media.

Es un tema que no indica un problema de investigación, hay una serie de conceptos y elementos empíricos implicados, la primera pregunta es ¿qué vemos y qué no vemos? Lo que vemos es un concepto muy general de exclusión social en el cual caben todas las expresiones que se refieren a estar fuera o dentro del conjunto de procesos, lo que vemos también es un modo morfológico de presentarse, definido a partir de datos e indicadores estandarizados, que dan lugar a clasificaciones usadas para las estadísticas censales, para medir los niveles de pobreza, tipo de vivienda, servicios, estudios de población, entre otros. Se observa una expresión de la realidad muy superficial. Existen datos y conceptos pero no hay problema.

El primer punto crítico se expresa cuando el sujeto ve el objeto desde su propia óptica o desde una lógica predeterminada; no solamente lo teórico sino lo empírico mismo correspondiente a una realidad preexistente que se ha acumulado como conceptos, como parte de la biografía intelectual del sujeto (ya conoce el objeto antes de aproximarse a él: la exclusión es un producto de la pobreza, ser pobre es ser excluido), y como datos estadísticos y descripciones cuantitativas.

Lo que no se ve es la posibilidad de apropiación de la realidad a partir de su reconstrucción, de su problematización. Vienen entonces los cuestionamientos: ¿De qué se habla cuando se habla de exclusión? ¿Solamente en la periferia de las ciudades medias se generan procesos de exclusión? El segundo punto crítico, que no se ve, es el problema de investigación. Es decir que el enunciado temático no ha sido cuestionado. Lo que no se ve es que sólo se tiene un enunciado sin contenidos propios. Por tanto, el conocimiento acumulado no tiene un significado concreto.

El tercer punto crítico es que el sujeto no se ha implicado, se mantiene como sujeto en potencia, en el sentido de no efectuar las necesarias rupturas epistemológicas, que la misma realidad impone para ser conocida. El objeto es una externalidad que existe de por-sí y sólo es necesario corroborarlo. No se ve que, 
como dice Zemelman, "la crítica debe reconocer como punto de partida la problematización para reconocer lo oculto... entre los requerimientos a los cuales hacemos referencia cabe señalar uno fundamental: la incorporación efectiva del sujeto" (Zemelman 2005:115) de tal manera que el fenómeno enunciado no ha sido reconstruido y apropiado como una realidad inteligible, por parte del sujeto.

Problematizar también significa definir lo dado en el contexto del fluir constante de la realidad, lo dándose de la realidad. Así el enunciado temático no revela ese recorte. No hay un cuestionamiento que permita identificar los problemas de investigación. No se ha revelado aún nada de la realidad, "no se ha reconocido lo oculto".

Un paso básico y necesario para construir el problema de investigación es la revisión crítica de la teoría; o sea repensar los conceptos en su capacidad de tener un significado o resignificarlos en función de la realidad sobre la que el sujeto reflexiona. Reconstruir la realidad teóricamente a partir del modo morfológico de presentarse el fenómeno.

Hacer un recorte de la realidad para definir lo dado, supone delimitar tanto teórica como empíricamente al fenómeno. De tal manera que en el tema enunciado: la exclusión social representa la posibilidad de construir una categoría, en la medida que a partir de ella se desprenden y agrupan conceptos de una mayor especificidad. La exclusión social entendida en lo general, como un proceso de integración-exclusión; exclusión-pobreza, carece de fuerza explicativa; es una aproximación dicotómica, y por ello mecánica. Pensar en la categoría de exclusión es pensar en una diversidad de conceptos dentro de la sociedad capitalista globalizada que ha generado nuevas formas de exclusión en diversos campos del quehacer social.

Lo anterior pone al sujeto frente a la exigencia de resignificar los procesos de exclusión y sus modos morfológicos de presentarse; y sólo lo puede hacer a partir de problematizar lo dado. Esta reflexión lleva a reconstruir conceptos específicos de la categoría de exclusión: laboral, racial, educativa, étnica, en la salud, sexual y política, entre otras diversas formas de manifestarse la exclusión. Conceptos que a su vez tienen que ser reconstruidos a partir de problemas concretos; generar la teoría partir del objeto y no poner la teoría acumulada en el objeto.

De esta manera es posible avanzar en la problematización. Ya no se trata de una cuestión preexistente, sino del horizonte que se abre para la construcción de la estructura sintética del objeto. El sujeto reflexiona y ya hay un sesgo, que sólo mediante el proceso de objetivación del objeto se puede minimizar, aunque siempre quedará la marca al momento de hacer el recorte de la realidad, apropiarse de lo dado elegido por él. Esta misma elección implica ya, por un lado, cierta sobredeterminación del sujeto sobre el objeto, desde el momento en que se efectúa un recorte espacio-temporal en un sentido orientado por el propio interés investigativo del sujeto, lo que el propio sujeto tendrá que superar a través del rompimiento-reorganización de su pensamiento, objetivar la subjetividad para plantear la realidad como un problema de conocimiento y no como un prejuicio; y por otro, la propia delimitación y la posibilidad de plantear el problema de investigación.

Como consecuencia de problematizar sobre el tema, se plantea como problema de investigación conocer la noción que se tiene sobre exclusión política en las colonias periféricas de las ciudades medias en México, frente a los procesos electorales del año 2009. Hay un recorte de la realidad, una primera aprehensión de lo dado y hay una exigencia de razonamiento en este planteamiento; no sólo se trata de definir los puntos de observación, los contenidos teóricos y empíricos, sino que el sujeto, como una exigencia epistemológica, 
tendrá que reflexionar sobre cómo pensar la realidad que ha construido y expresado en un problema de investigación. Aquí el sujeto se enfrenta a una doble ruptura epistemológica: romper con los métodos y teorías preexistentes y con los condicionamientos ideológicos derivados de simpatías, prácticas o influencias institucionales y a partir de ahí reorganizar el pensamiento.

Otra doble ruptura epistemológica necesaria se opera cuando el sujeto y su temporalidad se articulan con la temporalidad del objeto y luego rompe con las temporalidades externas tanto del sujeto como del objeto. Es decir, ¿corresponden los calendarios electorales con la noción de exclusión política de los grupos e individuos que integran las comunidades periféricas de las ciudades? Por esta razón es necesario reflexionar sobre las diversas temporalidades sociales y políticas con las que se entrecruzan las del objeto y del sujeto. Una temporalidad en la que el objeto se muestra y otra articulada con el fluir, con lo dándose de la realidad. Con esta lógica de la temporalidad del objeto tendrá que articularse la temporalidad del sujeto.

De esta forma se avanza en la construcción de la estructura sintética del objeto. Los conceptos expresan articulaciones de los procesos; corresponden a una apropiación teórica de la realidad que permite su reconstrucción empírica, no desde su aspecto aparente únicamente, sino desde la propia causa, la esencia de sus manifestaciones morfológicas. De tal forma que el concepto exclusión política debe dar cuenta de las características teóricas y empíricas del objeto.

El concepto implica dos categorías complejas, en ese sentido la reconstrucción es, igualmente, a partir del objeto. Igual que la categoría de exclusión, la política da cuenta de un campo de relaciones heterogéneas y diferenciadas; es decir ambas categorías dan cuenta de relaciones y no de efectos. Dicho de otra manera, tanto la exclusión como la política, dan cuenta de campos de relaciones, de procesos desiguales, ambas son producto de relaciones de poder. El concepto exclusión política permite reconocer relaciones y procesos con diferentes modos morfológicos de presentarse que pueden dar lugar a teorizaciones específicas.

Las relaciones y el ejercicio del poder político articulan ambas categorías y dan pie para construir el concepto exclusión política. La exclusión se refiere a procesos generados a partir de desigualdad, la inequidad y la dominación; la política tiene que ver con la confrontación de intereses y la dominación a través del ejercicio del poder. Las formas de manifestarse son producto de estas relaciones. El concepto exclusión política debe permitir la explicación teórica y empírica del objeto.

Con la construcción de las distintas determinantes y articulaciones de los planos de la realidad y de los elementos que interactúan para hacer aparecer lo dado como tal y no de otra manera, se constituye la estructura sintética del objeto de estudio. Este está presentado como totalidad concreta, como producto de las múltiples determinaciones e interacciones tanto de lo dado como de sus relaciones con lo dándose.

Aunque el itinerario no es lineal ni mecánico, lo hasta aquí expuesto es una posible base para la construcción de indicadores. Desde esta óptica es posible no sólo cuantificar sino también medir cualidades del objeto. Un procedimiento, entre otros, para la construcción de indicadores se daría de acuerdo a la siguiente lógica: Una vez construidos los contenidos teóricos del objeto en un proceso concreto-abstractoconcreto, es posible determinar los indicadores a partir de la operación de los conceptos. En el ejemplo propuesto habría que hacer operativo el concepto de exclusión política, para construir los indicadores que den cuenta del modo morfológico de presentarse el fenómeno, según la lógica del objeto. 
La exclusión política es parte de un campo y producto de un conjunto de relaciones heterogéneas y desiguales; la exigencia de razonamiento se orienta a pensar este campo a partir de las relaciones concretas que entablen los individuos o grupos frente al ejercicio y la estructura del poder. De esta manera, la exclusión política puede entenderse, en términos operativos, como la expresión de la desigualdad y la subordinación al poder, estar fuera o no pertenecer a(l) grupo(s) dominantes en cualquier nivel, a la estructura del poder político y al interior de la propia estructura de los grupos; se manifiesta en la separación entre sujetos, grupos e individuos y la estructura política. Los sectores grupos e individuos pueden no considerarse excluidos y ser excluyentes o pueden también, sentirse autoexcluidos. El discurso político dominante legitima tales condiciones. La exclusión política puede darse con respecto a: la información, el universo simbólico, los sujetos políticos (partidos, organizaciones empresariales, iglesias, sindicatos, organizaciones civiles, grupos de poder en la comunidad), a la gestión de gobierno, a las políticas públicas, a las instituciones electorales, entre otras instancias relacionadas con el ejercicio del poder en cualquier nivel.

Las manifestaciones empíricas de la exclusión política pueden manifestarse y agruparse en tres categorías que articulan un conjunto de indicadores. Tanto unos como otros pueden analizarse individual o relacionalmente. No necesariamente se presentan en estado puro. Las categorías y los indicadores son:

Autoexclusión: Representada por todas las formas que se asumen por parte de los grupos e individuos con respecto a la desigualdad y la subordinación. Estas formas se pueden presentar de manera crítica o pasiva y expresan posiciones correspondientes frente al discurso dominante.

Los indicadores que corresponden a esta categoría son: no participación, falta de interés, abstención, credibilidad, desinformación, valoraciones negativas de lo político, actitud crítica, conocimientodesinformación, entre otros.

Exclusión oficial: Representada por la imposición-aceptación del discurso dominante, que expresa la desigualdad y la subordinación como forma de estar fuera, la no pertenencia y la resignación frente al poder. Los individuos pueden no sentirse afectados por esta forma de exclusión política.

Los indicadores que definen esta categoría son: conocimiento, manipulación, engaño, desinterés, imposición de valoraciones, credibilidad, comunicación distorsionada, aceptación de la no pertenencia, entre otros indicadores.

Uso de la exclusión: representada por las formas de conciencia de la desigualdad y la subordinación y su uso a favor del individuo o del grupo. Significa la estructuración de respuestas al discurso dominante y permite obtener favores, apoyos, aceptación, status, ventajas y prebendas. También puede expresarse a través de formas de organización y participación y expresar posiciones críticas entre otras.

Los indicadores de esta categoría son: conveniencia, comodidad, compromiso, status, valoraciones, sobre vivencia política, formas de organización y formas de participación.

Se entiende que así como se definen operativamente las categorías tendría que procederse a hacer lo mismo con cada indicador, de tal manera que cada uno tiene un referente operativo sobre el cual puede teorizarse. Los indicadores constituyen las unidades de análisis que dan cuenta empírica del objeto de estudio. 
A partir de éstos viene la parte de aplicación de las metodologías seleccionadas; una vez que se sabe que los indicadores empíricos representan cualidades abstractas del objeto de estudio y que son estos los que se van a confrontar o medir a través de las metodologías estructuradas a partir de los indicadores. La determinación y el uso de metodologías cualitativas y/o cuantitativas es el resultado de este proceso de construcción de indicadores.

Como se ha expuesto, la construcción de indicadores no constituye un problema epistemológico menor, todo lo contrario, requiere de la exigencia de un modo crítico de reflexionar, de apropiarse y reconstruir la realidad; requiere de vigilancia, y permanentes rupturas y sus correspondientes formas de reorganizar el pensamiento.

\section{Bibliografía}

Bachelard, G. 1998. El compromiso racionalista. México: Siglo XXI.

Bachelard, G. 1999. La formación del espíritu científico. México: Siglo XXI.

Boudon, R. y Lazarsfeld, P. 1985. Metodología de las ciencias sociales. Barcelona: Laila.

Bourdieu, P. 2003. El oficio del científico. Barcelona: Anagrama.

Elster, J. 2000. Tuercas y tornillos. Barcelona: Gedisa.

Husserl, E. 2005. Meditaciones cartesianas. México: FCE.

Kosik, K. 1967. Dialéctica de lo concreto. México: Grijalbo.

Lecourt, D. 1978. Para una crítica de la epistemología. México: Siglo XXI.

Mills Wright, C. 2000. La imaginación sociológica. México: FCE.

Pasquino, G. (Ed). 1996. Manual de ciencia política. Madrid: Alianza Universidad.

Sacristán, M. 1988. Antología de Antonio Gramsci. México: Siglo XXI.

Sánchez, I. y Sosa, R. 2004. América latina: los desafíos del pensamiento crítico. México: Siglo XXI-UNAM.

Schutz, A. y Luckmann, T. 1973. Las estructuras del mundo de la vida. Buenos Aires: Amorrortu.

VV.AA. (1994) Círculos de Reflexión Latinoamericana en Ciencias Sociales: Cuestiones de Teoría y Método. Suplemento 45 Revista Anthropos. Barcelona: España

Zemelman, H. 1989. Crítica epistemológica de los indicadores. Jornadas 114. México: El Colegio de México.

Zemelman, H. 2005. Voluntad de conocer, el sujeto y su pensamiento en el paradigma crítico. México: Anthropos, IPECAL-UNACH.

Recibido el 12 Dic 2008

Aceptado el 23 Feb 2009 